\title{
Science and Technology: An Aid in Building Human Resilience
}

\author{
Dr. Sheeba Himani Sharma \\ Associate Professor \\ Department of English \\ St. Andrew's College \\ Gorakhpur, India \\ sheebahimani1287@gmail.com
}

\begin{abstract}
Since inception, human race has always witnessed pandemics, disasters and wars, but it is the resilient spirit that has brought us this far. With each passing devastation, we rebuild ourselves and stand even stronger than before. Although there are numerous factors that help to inculcate resilience within humans, Science and Technology have ever remained faithful and have suggested promising ways using which we could combat our 'unseen enemy' today and even in the past. Today, at this hour, when the world is overburdened by the chaos of COVID-19, people are looking up to Science and Technology for the ways they can offer to eradicate this disease from the face of the earth. As it is rightly said, "Necessity is the mother of invention", it can be safely concluded that Science and Technology are boosted by resilience. The human race has found the best of its innovations while undergoing some very troubled times. The current crisis in front of us is the situation now where the world has been segregated and people have been isolated. But thanks to technology that is knitting us close together. On the other hand, Science is playing its part to help discover effective drugs, improve human immunity and all this is being done to ensure the smooth running of human civilization. This paper intends to bring into view the notable contributions of Science and
\end{abstract}


Technology that are today being used and exploited extensively in light of this pandemic outbreak.

Keywords: Human, resilience, science, technology.

Despite being time and again severely devastated, suffering major setbacks and most recently the whole world being in clutches for months due to the rise of the pandemicCOVID-19, (caused by SARS-CoV-2 or the Novel Coronavirus), there is a zeal in the human race to fight back all odds and to restore the lifestyle back to its usual. Ever since the evolution of mankind on the face of this earth to the present day, the resilient spirit of man has brought him thus far. This is a God-gifted trait in humans that instills in them a desire to stand out, face the challenges no matter how crushed or ruined the condition be.

Whenever we look back to the history of the pandemics that have hit the human race, Science has always come forward as a faithful friend to fight it and has suggested promising ways using which we could combat them in the past. Science has always helped to build resilience in humans whenever they were in a situation of crisis. Even today, at this hour, when the world is overburdened by the chaos of COVID-19, people are looking up to Science and Technology for the ways they can offer to eradicate this disease from the face of the earth.

Now the question arises as to what Science and Technology has to offer to humankind so as to make them resilient in this critical situation. As it is rightly said "Necessity is the mother of invention", it can be safely concluded that technology is boosted by resilience. The human race has found the best of its innovations while undergoing some very trouble times. Science, on the other hand, stands independent and hence proves to be the foundation stone upon which all our further study is based. 
Even the most basic knowledge that we have acquired about immunology, has taken its form based on the research that took place centuries ago, which involve the study of the response of the human immune system to various pathogens. Most of the understanding that has been developed till now has such benefits that were unimaginable when the research was then being conducted. Many of today's indispensable medical interventions are rooted in the fundamental discoveries made at the frontiers of the physical world. Ever since the outbreak of COVID-19, the world is already witnessing a wide swath of 'curiosity-driven research'. On studying the dynamics of the Bat which is supposed to be the carrier of the Human Corona Virus, the scientists have come to a better understanding as to how this virus transmits. The Human Genome Project, that began as a scientific endeavor back in the 1990, led to the DNA and RNA sequencing that enabled the scientists working in that area to determine the evolution of COVID-19, keeping in view the long term immunity that could be developed against it.

As the news of increase in the number of patients suffering with an unknown pneumonia fever reached the World Health Organization (WHO), it declared that it was an RNA virus which belonged to the family Coronaviridae and closely related to those viruses which caused the Severe Acute Respiratory Syndrome (SARS). It was soon found out that the carrier of this virus was an animal i.e., bat and was transferred from it to humans. This virus has RNA as its genome and it contains of an envelope made up of molecules called lipoproteins. Besides this, it also contains three other proteins, namely, the spike (S) protein which imparts it a crown like or spiked structure, hence the name Coronavirus ("corona" stands for "crown" in Latin), the envelope (E) protein and the membrane (M) protein. If this virus is present anywhere on the skin (outside the human body), this envelope of proteins can be broken by the use of soap, thus rendering it ineffective. It is for this reason that it is advised by the scientists and doctors to frequently wash hands. 
Then the next major focus of the study was to determine how the virus spreads, knowing which its spread could be curbed. The epidemiological studies along with the clinical data showed that the virus spreads from person to person either through direct contact or through the droplets of a patient's cough or nasal discharge. Hence, the idea of "physical distancing" was resorted to limit the outbreak of the disease.

Owing to the rapid spread of COVID-19, there was a need for accurate diagnosis and rapid identification of the virus and the research was therefore accelerated towards understanding the structure, molecular biology, and immunology of SARS-CoV-2. In this regard, several test have been reported across the globe. FDA has already approved diagnostics tests that are capable of detecting antibodies specific to SARS- CoV-2. Some other diagnostic tests are the Polymerase Chain Reaction (PCR) based technique and the Next-Generation Sequencing.

A study has indicated that a protein $\mathrm{TMPRSS}_{2}$ assists the virus in entering the host cell. It also showed that a synthetic inhibitor, Camostat Mesylate, attacks this protein, thus blocking the entry of the virus in the host cell. This research can give a breakthrough finding out a suitable drug for Coronavirus. The researchers have also showed that the virus reproducing itself inside the cells could be blocked using an inhibitor, $\alpha$-ketoamide, thus offering a promising strategy for potential therapeutic intervention. The available drugs are being repurposed and being tested for their efficacy against COVID-19. The scientists are also targeting their study to the groups of people who are naturally resistant to the virus and aiming at the comparative gene sequencing of such people with the COVID-19 susceptible patients. This might assist in effective drug development against the virus.

As with the onset of the lockdown and the havoc caused by the spreading of the disease, there are other major issues that can be seen in the society. These issues have deeply influenced the psychology and behavior of the people in a very negative way. The 
Mathematicians have been modeling various functions that include the epidemiology of disease spread or the social psychology of human behavior. This information serves as a necessary foundation using which optimal recommendations could be put forward in order to determine the course of actions that should be taken.

Science doesn't work on its own until and unless the theories put forward by it are devised for human use through technology. The current crisis in front of us is the situation now where the world has been segregated and people have been isolated. But thanks to technology that is knitting us close together. People no more feel lonely or are in a state of a dilemma of the whereabouts and welfare of their loved ones. Easy and fastest communication is at the tip of our fingers. Despite being quite advanced, technology is still being upgraded and modified to cope up with the need of the hour.

One of the greatest boons technology has offered is the expansive use of digital platform in every area life. To start with, let us give a moment of thought to the various apps that have been developed strategically and specially in regards to COVID-19, wherein the scientists are being helped in an enormous way by the valuable information provided at individual level. This information could be highly useful to fight the dreaded virus. There are numerous portfolios built which come together on a single platform wherein the person can fill his/her details, the symptoms they show, their travel history as well as their medical history. It would be notable to mention here of the Arogya Setu app promoted by the Honorable Prime Minister of India, Sri Narendra Modi, himself, which is being used extensively in India as the biggest source information from among the masses. By using these smartphone apps, the information gathered can contribute significantly to the real time data and can be put to action immediately. In the words of Dr. Steven Steinhubl, the director of digital medicine at the Scripps Research Translational Institute, "This format provides much more pragmatic and real world information, so it is much more immediately actionable." 
As we proceed further, we find that during the last few years, technology has witnessed a boost in terms of medical equipments that are now proving to be of a great help dealing with SARS-CoV-2. The equipments that have been developed lately are finding much use in the present day situation. The information recorded below has been taken from the April issue of The Economic Times.

1. Bigtec Labs, Bengaluru, has a subsidiary branch Molbio that has developed test kits for clinical diagnosis, that gives accurate results in about an hour. These kits have been made available in the market at a very cheap price of ₹1340.

2. The centralized air conditioner system was a problem for the COVID-19 patients as the virus can easily travel through the air ducts. To deal with this situation, a novel technique developed by AgVa Healthcare, Noida, in 2017, is now finding importance. The company developed portable ventilators that run using room air without the need of compressed air. Most interesting fact is that their prices are almost one-fifth of those that are imported.

3. Mylab Discovery Solutions, Pune, developed India's first RT-PCR (Real Time Reverse Transcription Polymerase Chain Reaction) testing kits within six weeks of the onset of COVID-19. In March-end, these kits got commercial approval and they are priced at ₹1200 per test.

4. The Institute of Genomics and Integrative Biology (IGIB), which has done the Human Genome Sequencing, is now collaborating with national bodies such as Indian Council of Medical Research (ICMR) and National Centre for Disease Control (NCDC) and also with multinational corporations such as Intel to study human-virus interaction, their response to drugs and to study about the side effects caused by these drugs, if any.

Conclusion: 
In the words of Kaushik Biswas, Associate Professor at the Division of Molecular Medicine, Bose Institute, Kolkata,"This COVID-19 pandemic has brought to our attention how building up scientific temperament and propagation of scientific culture are critically important to tackling a pandemic of this scale. The knowledge so acquired can help us understand the very basics of the disease process, helping us make progress in treating and preventing it."

The medical establishment, the healthcare industry and the resource givers to thousands of hundreds of professionals are now working on the front-line of the "battlefield" and are using the full force their knowledge and manoeuvre to stem this pervasive disease. Although it cannot be predicted by anyone which venture by the scientists will prove to be fruitful at the earliest, researches, studies and tests would still be pursued in order to offer the best and safe environment to people round the globe. All we can do on our part is to be an attentive listener, respecting and following the advices of our professionals and playing an active part in spreading awareness to those from whom education, social media and other means are deprived and strive together, hand in hand, to make the world a better place to live in. On the other hand, Science and Technology always have and will continue in helping build resilience in humans in any such time of crisis.

The fact remains that there is an entire Universe from which we can learn and most of the lessons we learn from venturing into one situation often leads us to those applications that would be later benefitting us. Therefore, we should not be hesitant in taking a few daring steps to deal with the current crisis but should have the full knowledge that whatever maybe the result, it will instill in us a spirit of courage and and will boost the strength within us.

These necessity-driven innovations target upon the importance of nurturing a scientific community through gradual and sustained development and also how the propagation of a scientific environment is critically important towards becoming a 
"Pandemic-ready" nation in the future. If we want to be well equipped and prepared to face the threats that humanity will face in the coming years, we need to now start investing our efforts positively into inculcating a scientific temperament within us and including ourselves at the fundamental level and not only leaving this responsibility on the front-line workers i.e., the scientists, doctors and nurses etc. Even though we are facing bitter consequences of the virus and this pandemic has hit us hard, it is now our turn to demonstrate how scientific and logical we are in our approach. Let us be prepared with curious minds which ultimately leads to knowledge and understanding and prove how far we have come together as a civilization.

I would like to conclude with a few lines of the poem, "A Psalm of Life" by Henry Wadsworth Longfellow:

Let us, then, be up and doing,

With a heart for any fate;

Still achieving, still pursuing,

Learn to labor and to wait. 


\section{Works Cited}

The 3 Ways Science Will Get Us Through The COVID-19 Pandemic www.forbes.com/sites/startswithabang/2020/04/07/the-3-ways-science-will-get-usthrough-the-covid-19-pandemic/\#1e204cf62fc3

Can Coronavirus Crisis change Indian science for good? Www.economictimes.indiatimes.com/news/science/can-coronavirus-crisis-changeindian-science-for-good/articleshow/75224904.cms

The role of science and scientists in mitigating the COVID-19 pandemic- Part 1 www./indiabioscience.org/columns/opinion/the-role-of-science-and-scientists-inmitigating-the-covid-19-pandemic

The role of science and scientists in mitigating the COVID-19 pandemic- Part 2 www./indiabioscience.org/columns/opinion/the-role-of-science-and-scientists-inmitigating-the-covid-19-pandemic-part-2 